\title{
A COMPARISON AND ERROR ANALYSIS OF ERROR BOUNDS
}

\author{
A. R. KASHIF ${ }^{1}$, T. S. KHAN ${ }^{2}$, A. QAYYUM ${ }^{3, *}$, AND I. FAYE $^{4}$ \\ ${ }^{1}$ Department of Mathematics, Capital University of Science $\& 3$ Technology Islamabad, Pakistan \\ ${ }^{2}$ Department of Mathematics, University of Peshawar, Peshawar, Pakistan \\ ${ }^{3}$ Department of Mathematics, University of Hail, 2440, Saudi Arabia \\ ${ }^{4}$ Fundamental and Applied Sciences Department, Universiti Teknologi Petronas, Malaysia \\ ${ }^{*}$ Corresponding author: atherqayyum@gmail.com
}

\begin{abstract}
In this paper, we present an error analysis with the help of Ostrowski type inequalities for $n$-times differentiable mappings by using $n$-times peano kernel. A comparison is also presented which shows that obtained error bounds are better than the previous error bounds.
\end{abstract}

\section{IntRoduction}

Integral inequalities have many potential applications in many practical problems of the real world. Theory of integral inequalities is rapidly growing with the help of some basic tools of functional analysis, topology and fixed point theory. Ostrowski inequality is one of them, that can be defined as: Estimate the deviation of functional value from its average value and the estimation of approximating area under the curve. In the last few years, many researchers tried to obtain better bounds of Ostrowski inequality in the form of different Lebesgue spaces. (see for instance [16]- [19]). In many practical problems, it is important to bound one quantity by another quantity. The classical inequalities such as Ostrowski are very useful for this purpose.

Received 2018-02-13; accepted 2018-04-27; published 2018-09-05.

2010 Mathematics Subject Classification. 00A00.

Key words and phrases. Ostrowski inequality; Čebyŝev-Grüss inequality and Čebyŝev functional.

(C) 2018 Authors retain the copyrights of their papers, and all open access articles are distributed under the terms of the Creative Commons Attribution License. 
To make new Ostrowski type inequalities, Peano kernel is the most important tool. With the help of different Peano kernels, different types of Ostrowski type inequalities can be obtained. Efficiency of quadrature rules also depend on the selection of kernel. An analysis with the help of some graphs are also shown. At the end, a comparison with the previous results is also presented.

In 1938, Ostrowski [15] discovered the following useful integral inequality.

Theorem 1.1. Let $f:[a, b] \rightarrow \mathbb{R}$ be continuous on $[a, b]$ and differentiable on $(a, b)$, whose derivative $f^{\prime}:(a, b) \rightarrow \mathbb{R}$ is bounded on $(a, b)$, i.e.

$$
\left\|f^{\prime}\right\|_{\infty}=\sup _{t \in[a, b]}\left|f^{\prime}(t)\right|<\infty
$$

then for all $x \in[a, b]$

$$
\left|f(x)-\frac{1}{b-a} \int_{a}^{b} f(t) d t\right| \leq\left[\frac{1}{4}+\left(\frac{x-\frac{a+b}{2}}{b-a}\right)^{2}\right](b-a)\left\|f^{\prime}\right\|_{\infty} .
$$

We mention another inequality called Grüss inequality [13] which is stated as the integral inequality that establishes a connection between the integral of the product of two functions and the product of the integrals, which is given below.

$$
\begin{aligned}
& \left|\frac{1}{b-a} \int_{a}^{b} f(x) g(x) d x-\frac{1}{b-a} \int_{a}^{b} f(x) d x \cdot \frac{1}{b-a} \int_{a}^{b} g(x) d x\right| \\
\leq & \frac{1}{4}(\Phi-\varphi)(\Gamma-\gamma),
\end{aligned}
$$

where

$$
\varphi \leq f(x) \leq \Phi \text { and } \gamma \leq g(x) \leq \Gamma,
$$

for all $x \in[a, b]$. The constant $\frac{1}{4}$ is sharp in (1.2) .

In [8], Dragomir and Wang combined Ostrowski and Grüss inequality to give a new inequality which they named Ostrowski-Grüss type inequalities.

In [9], Guessab and Schmeisser proved the following Ostrowski's inequality:

Theorem 1.2. Let $f:[a, b] \rightarrow \mathbb{R}$ satisfy the Lipschitz condition i.e., $|f(t)-f(s)| \leq M|t-s|$. Then for all $x \in\left[a, \frac{a+b}{2}\right]$, we have

$$
\left|\frac{f(x)+f(a+b-x)}{2}-\frac{1}{b-a} \int_{a}^{b} f(t) d t\right| \leq\left[\frac{1}{8}+2\left(\frac{x-\frac{3 a+b}{4}}{b-a}\right)^{2}\right](b-a) M .
$$

In (1.3), the point $x=\frac{3 a+b}{4}$ yields the following trapezoid type inequality.

$$
\left|\frac{f\left(\frac{3 a+b}{4}\right)+f\left(\frac{a+3 b}{4}\right)}{2}-\frac{1}{b-a} \int_{a}^{b} f(t) d t\right| \leq \frac{b-a}{8} M .
$$

The constant $\frac{1}{8}$ is sharp in (1.4). 
In [4], Barnett et.al proved some Ostrowski and generalized trapezoid inequalities. Dragomir [7] and Liu [10] established some companions of ostrowski type integral inequalities. Alomari [1] proved the following inequality:

Let $f:[a, b] \rightarrow \mathbb{R}$ be a differentiable mapping on $(a, b)$. If $f^{\prime} \in L^{1}[a, b]$ and $\gamma \leq f^{\prime}(t) \leq \Gamma$, for all $t \in[a, b]$, then

$$
\left|\frac{f(x)+f(a+b-x)}{2}-\frac{1}{b-a} \int_{a}^{b} f(t) d t\right| \leq \frac{1}{8}(b-a)(\Gamma-\gamma) .
$$

Recently, Liu [11] and Liu et.al [12] proved some ostrowski type inequalities. In all references mentioned above, authors proved their results by using kernels with two or three steps.

Qayyum et. al [18] presented a refinements of Ostrowski Inequality for $n$-th differentiable functions as:

Define $n$-times Peano kernel $P(x,):.[a, b] \rightarrow \mathbb{R}$ by

$$
P_{n}(x, t)= \begin{cases}\frac{1}{n !}(t-a)^{n}, & t \in\left(a, \frac{a+x}{2}\right] \\ \frac{1}{n !}\left(t-\frac{3 a+b}{4}\right)^{n}, & t \in\left(\frac{a+x}{2}, x\right] \\ \frac{1}{n !}\left(t-\frac{a+b}{2}\right)^{n}, & t \in(x, a+b-x] \\ \frac{1}{n !}\left(t-\frac{a+3 b}{4}\right)^{n}, & t \in\left(a+b-x, \frac{a+2 b-x}{2}\right] \\ \frac{1}{n !}(t-b)^{n}, & t \in\left(\frac{a+2 b-x}{2}, b\right],\end{cases}
$$

for all $x \in\left[a, \frac{a+b}{2}\right]$,then following lemma holds:

Lemma 1.1. Let $f:[a, b] \rightarrow \mathbb{R}$ be an n-times differentiable function such that $f^{(n-1)}(x)$ for $n \in N$ is absolutely continuous on $[a, b]$ then

$$
\frac{1}{b-a} \int_{a}^{b} P_{n}(x, t) f^{(n)}(t) d t
$$




$$
\begin{aligned}
& =\sum_{k=0}^{n-1} \frac{(-1)^{n+k+1}}{(k+1) !} \\
& \times\left[\frac{1}{2^{k+1}}\left\{(x-a)^{k+1}-\left(x-\frac{a+b}{2}\right)^{k+1}\right\} f^{(k)}\left(\frac{a+x}{2}\right)\right. \\
& +\left\{\left(x-\frac{3 a+b}{4}\right)^{k+1}-\left(x-\frac{a+b}{2}\right)^{k+1}\right\} f^{(k)}(x) \\
& +(-1)^{k+1}\left\{\left(x-\frac{a+b}{2}\right)^{k+1}-\left(x-\frac{3 a+b}{4}\right)^{k+1}\right\} f^{(k)}(a+b-x) \\
& \left.+\left(\frac{-1}{2}\right)^{k+1}\left\{\left(x-\frac{a+b}{2}\right)^{k+1}-(x-a)^{k+1}\right\} f^{(k)}\left(\frac{a+2 b-x}{2}\right)\right] \\
& +\frac{(-1)^{n}}{b-a} \int_{a}^{b} f(t) d t
\end{aligned}
$$

for all $x \in\left[a, \frac{a+b}{2}\right]$.

\section{MAIN RESUlts}

2.1. Integral inequalities for $\left\|f^{(n)}\right\|_{\infty}$.

Theorem 2.1. Let $f:[a ; b] \rightarrow R$ be an $n$-times differentiable function such that $f^{(n-1)}(x)$ for $n \in N$ on $(a, b)$ is absolutely continuous on $[a, b]$, then

$$
\begin{aligned}
& \mid \int_{a}^{b} f(t) d t-(b-a) \sum_{k=0}^{n-1} \frac{(-1)^{k}}{(k+1) !}\left[\frac{1}{2^{k+1}}\left\{(x-a)^{k+1}-\left(x-\frac{a+b}{2}\right)^{k+1}\right\}\right. \\
& \times f^{(k)}\left(\frac{a+x}{2}\right)+\left\{\left(x-\frac{3 a+b}{4}\right)^{k+1}-\left(x-\frac{a+b}{2}\right)^{k+1}\right\} f^{(k)}(x) \\
& +(-1)^{k+1}\left\{\left(x-\frac{a+b}{2}\right)^{k+1}-\left(x-\frac{3 a+b}{4}\right)^{k+1}\right\} f^{(k)}(a+b-x) \\
& \left.+\left(\frac{-1}{2}\right)^{k+1}\left\{\left(x-\frac{a+b}{2}\right)^{k+1}-(x-a)^{k+1}\right\} f^{(k)}\left(\frac{a+2 b-x}{2}\right)\right] \\
& \left.\leq \frac{\left\|f^{(n)}\right\|_{\infty}\left[\frac{1}{2^{n}}(x-a)^{n+1}+\left(1+(-1)^{n}\right)\left(x-\frac{3 a+b}{4}\right)^{n+1}\right.}{(n+1) !} \mid \frac{-1+(-1)^{n+1}}{2^{n+1}}-\left(1+(-1)^{n}\right)\right)\left(x-\frac{a+b}{2}\right)^{n+1}
\end{aligned}
$$

for all $x \in\left[a, \frac{a+b}{2}\right]$.

The following new quadrature rules can be obtained while investigating error bounds using above Theorem. 


$$
\begin{aligned}
& Q_{n, 1}(f):=\int_{a}^{b} f(t) d t \\
& \approx \sum_{k=0}^{n-1} \frac{(b-a)^{k+2}}{2^{k+1}(k+1) !}\left[f^{(k)}(a)+(-1)^{k} f^{(k)}(b)\right] \\
& +\left[f^{(n-1)}(b)-f^{(n-1)}(a)\right] \frac{(b-a)^{n}}{2^{n+1}(n+1) !}\left(1+(-1)^{n}\right) \\
& Q_{n, 2}(f):=\int_{a}^{b} f(t) d t \\
& \approx \sum_{k=0}^{n-1} \frac{(b-a)^{k+2}(-1)^{k}}{4^{k+1}(k+1) !}\left[f^{(k)}\left(\frac{3 a+b}{4}\right)\right. \\
& \left.+\left\{1+(-1)^{k}\right\} f^{(k)}\left(\frac{a+b}{2}\right)+(-1)^{k} f^{(k)}\left(\frac{a+3 b}{4}\right)\right] \\
& +\left(f^{(n-1)}(b)-f^{(n-1)}(a)\right) \times \frac{2}{4^{n+1}} \frac{(b-a)^{n}}{(n+1) !}\left((-1)^{n}+1\right) \\
& Q_{n, 3}(f):=\int_{a}^{b} f(t) d t \\
& \approx \sum_{k=0}^{n-1} \frac{(-1)^{k}}{(k+1) !} \frac{(b-a)^{k+2}}{4^{k+1}}\left[\frac{1+(-1)^{k}}{2^{k+1}}\left(f^{(k)}\left(\frac{7 a+b}{8}\right)+f^{(k)}\left(\frac{a+7 b}{8}\right)\right)\right. \\
& \left.+\left\{(-1)^{k} f^{(k)}\left(\frac{3 a+b}{4}\right)+f^{(k)}\left(\frac{a+3 b}{4}\right)\right\}\right] \\
& +\left[f^{(n-1)}(b)-f^{(n-1)}(a)\right] \frac{(b-a)^{n}}{4^{n+1}(n+1) !}\left((-1)^{n}+1\right)\left(\frac{1}{2^{n}}+1\right) .
\end{aligned}
$$

From [12], using Theorem 3.1, Cerone's Quadrature Rules

$$
\begin{gathered}
q_{n, 1}(f):=\int_{a}^{b} f(t) d t \\
\approx \sum_{k=0}^{n-1} \frac{(b-a)^{k+1}}{(k+1) !} f^{(k)}(a) \\
q_{n, 2}(f):=\int_{a}^{b} f(t) d t \\
\approx \sum_{k=0}^{n-1} \frac{(b-a)^{k+1}}{(k+1) !}\left(1+(-1)^{k}\right) f^{(k)}\left(\frac{a+b}{2}\right)
\end{gathered}
$$




$$
\begin{aligned}
& q_{n, 3}(f):=\int_{a}^{b} f(t) d t \\
& \approx \sum_{k=0}^{n-1} \frac{1}{(k+1) !} \frac{(b-a)^{k+1}}{4^{k+1}}\left((1)^{k+1}+(-1)^{k}\right) f^{(k)}\left(\frac{3 a+b}{4}\right)
\end{aligned}
$$

Now, we present a comparison between Cerone's error bouds with obtained error bounds.

\section{A Comparison and Error Analysis of Error Bounds}

From [6], Cerone's error bounds are given:

$$
\begin{aligned}
& E_{c, 1}=\frac{(b-a)^{n+1}}{(n+1) !} \\
& E_{c, 2}=\frac{(b-a)^{n+1}}{2^{n}(n+1) !} \\
& E_{c, 3}=\frac{(b-a)^{n+1}\left(1+3^{n+1}\right)}{4^{n+1}(n+1) !} .
\end{aligned}
$$

Our error bounds obtained from above Theorem, are given below:

$$
\begin{aligned}
E_{a, 1}= & \frac{(b-a)^{n+1}}{(n+1) !}\left[\frac{1}{2^{n+1}}+\frac{(-1)^{n}+1}{2^{n+1}}\right] \\
E_{a, 2}= & \frac{(b-a)^{n+1}}{2^{n}(n+1) !}\left[\frac{1}{2^{n+3}}+\frac{(-1)^{n}+1}{2^{3 n+4}}\right] \\
E_{a, 3}= & \frac{(b-a)^{n+1}\left(1+3^{n+1}\right)}{4^{n+1}(n+1) !} \\
& \times\left[\frac{(-1)^{n}+1}{1+3^{3 n+1}}+\frac{(-1)^{n}+1}{2^{n+1}\left(1+3^{3 n+1}\right)}+\frac{3}{2^{n+1}\left(1+3^{3 n+1}\right)}\right]
\end{aligned}
$$

To show that our error bounds are less than Cerone's error bound, we have to show that

$$
\begin{gathered}
\frac{1}{2^{n+1}}+\frac{(-1)^{n}+1}{2^{n+1}}<1 \\
\frac{1}{2^{n+3}}+\frac{(-1)^{n}+1}{2^{3 n+4}}<1 \\
\frac{(-1)^{n}+1}{1+3^{3 n+1}}+\frac{(-1)^{n}+1}{2^{n+1}\left(1+3^{3 n+1}\right)}+\frac{3}{2^{n+1}\left(1+3^{3 n+1}\right)}<1
\end{gathered}
$$

Case 1 First we will prove that

$$
\frac{1}{2^{n+1}}+\frac{(-1)^{n}+1}{2^{n+1}}<1
$$

Since

$$
2^{n}>1 \quad \forall n \in\{1,2,3, \ldots\}
$$


Also

$$
\begin{array}{rr}
2^{n+1}>2 & \forall n \in\{1,2,3, \ldots .\} \\
\Longrightarrow \frac{1}{2^{n+1}}<\frac{1}{2} \quad \forall n \in\{1,2,3, \ldots .\}
\end{array}
$$

Now, since

$$
\frac{1+(-1)^{n}}{2^{n+1}}= \begin{cases}0, & \text { if } n \text { is odd } \\ \frac{1}{2^{n}}, & \text { if } n \text { is even }\end{cases}
$$

And

$$
\begin{aligned}
\frac{1}{2^{n+1}}+\frac{1+(-1)^{n}}{2^{n+1}} & <\frac{1}{2} \\
& <1 \quad \forall \text { odd } n
\end{aligned}
$$

Now

$$
\begin{aligned}
& 2^{n+1}>4 \\
& \frac{1}{2^{n+1}}<\frac{1}{4} \quad \forall \text { even } n
\end{aligned}
$$

Therefore,

$$
\begin{aligned}
\frac{1}{2^{n+1}}+\frac{1+(-1)^{n}}{2^{n+1}} & <\frac{1}{4}+\frac{1}{2^{n}} \\
& <\frac{1}{4}+\frac{1}{2} \\
& <1
\end{aligned}
$$

Hence proved that

$$
\frac{1}{2^{n+1}}+\frac{(-1)^{n}+1}{2^{n+1}}<1 \quad \forall n \in\{1,2,3, \ldots .\} .
$$

Case 2 Now, we will prove that

$$
\frac{1}{2^{n+3}}+\frac{(-1)^{n}+1}{2^{3 n+4}}<1
$$

Since

$$
2^{n}>1 \quad \forall n \in\{1,2,3, \ldots .\}
$$

Also

$$
\begin{aligned}
2^{n+3} & >2^{3} \\
\frac{1}{2^{n+3}} & <\frac{1}{8} \\
& <1
\end{aligned} \quad \forall n \in\{1,2,3, \ldots .\}
$$


Since

$$
\frac{1+(-1)^{n}}{2^{3 n+4}}= \begin{cases}0, & \text { if } n \text { is odd } \\ \frac{1}{2^{3 n+3}}, & \text { if } n \text { is even }\end{cases}
$$

And

$$
\frac{1}{2^{n+3}}+\frac{1+(-1)^{n}}{2^{3 n+4}}<1 \quad \forall \text { odd } n
$$

Now

$$
\begin{aligned}
2^{n+3}>2^{9} & \forall \text { even } n \\
\frac{1}{2^{3 n+4}}<\frac{1}{2^{9}} & \forall \text { even } n
\end{aligned}
$$

Therefore

$$
\begin{aligned}
\frac{1}{2^{n+3}}+\frac{1+(-1)^{n}}{2^{3 n+4}} & <\frac{1}{2^{9}}+\frac{1}{8} \\
& <\frac{1}{2}+\frac{1}{8} \\
& <1 \quad \forall \text { even } n
\end{aligned}
$$

Hence proved that

$$
\frac{1}{2^{n+3}}+\frac{(-1)^{n}+1}{2^{3 n+4}}<1 \quad \forall n \in\{1,2,3, \ldots .\} .
$$

Case 3 We will prove that

$$
\frac{(-1)^{n}+1}{1+3^{3 n+1}}+\frac{(-1)^{n}+1}{2^{n+1}\left(1+3^{3 n+1}\right)}+\frac{3}{2^{n+1}\left(1+3^{3 n+1}\right)}<1
$$

Since

$$
\begin{gathered}
\frac{1+(-1)^{n}}{1+3^{3 n+1}}= \begin{cases}0, & \text { if } n \text { is odd } \\
\frac{2}{1+3^{3 n+1}}, & \text { if } n \text { is even, }\end{cases} \\
\frac{1+(-1)^{n}}{2^{n+1}\left(1+3^{3 n+1}\right)}= \begin{cases}0, & \text { if } n \text { is odd } \\
\frac{1}{2^{n}\left(1+3^{3 n+1}\right)}, & \text { if } n \text { is even }\end{cases}
\end{gathered}
$$

Now

$$
\begin{aligned}
2^{n+1}\left(1+3^{3 n+1}\right) & >2^{n+1} \cdot 3^{3 n+1} \\
& >2^{2} \cdot 3^{4} \\
& >2 \cdot 3 \quad \forall \text { odd } n
\end{aligned}
$$

or

$$
\begin{aligned}
& \frac{1}{2^{n+1}\left(1+3^{3 n+1}\right)}<\frac{1}{6} \\
& \frac{3}{2^{n+1}\left(1+3^{3 n+1}\right)}<\frac{1}{2} \quad \forall \text { odd } n
\end{aligned}
$$


Therefore

$$
\begin{aligned}
\frac{1+(-1)^{n}}{1+3^{3 n+1}}+\frac{1+(-1)^{n}}{2^{n+1}\left(1+3^{3 n+1}\right)}+\frac{3}{2^{n+1}\left(1+3^{3 n+1}\right)} & <\frac{1}{2} \\
& <1
\end{aligned}
$$

Now

$$
\frac{1+(-1)^{n}}{1+3^{3 n+1}}=\frac{2}{1+3^{3 n+1}} \forall \text { even } n
$$

and

$$
\frac{1+(-1)^{n}}{2^{n+1}\left(1+3^{3 n+1}\right)}=\frac{1+(-1)^{n}}{2^{n}\left(1+3^{3 n+1}\right)} \quad \forall \text { even } n
$$

As we know that

$$
1+3^{3 n+1}>3^{3 n+1} \quad \forall \text { even } n
$$

or

$$
\begin{aligned}
\frac{1}{1+3^{3 n+1}} & <\frac{1}{3^{3 n+1}} \\
& <\frac{1}{3} \forall \text { even } n
\end{aligned}
$$

Again, we know that

$$
2^{n}\left(1+3^{3 n+1}\right)>2^{n} \cdot 3^{3 n+1} \forall \text { even } n
$$

or

$$
\begin{aligned}
\frac{1}{2^{n}\left(1+3^{3 n+1}\right)} & <\frac{1}{2^{n} \cdot 3^{3 n+1}} \\
& <\frac{1}{12} \quad \forall \text { even } n
\end{aligned}
$$

Now

$$
2^{n+1}\left(1+3^{3 n+1}\right)>2^{n+1} \cdot 3^{3 n+1}
$$

or

$$
\begin{aligned}
\frac{1}{2^{n+1}\left(1+3^{3 n+1}\right)} & <\frac{1}{2^{n+1} \cdot 3^{3 n+1}}<\frac{1}{24} \\
& <\frac{1}{8} \quad \forall \text { even } n
\end{aligned}
$$

Hence proved that

$$
\frac{1+(-1)^{n}}{1+3^{3 n+1}}+\frac{1+(-1)^{n}}{2^{n+1}\left(1+3^{3 n+1}\right)}+\frac{3}{2^{n+1}\left(1+3^{3 n+1}\right)}<1
$$




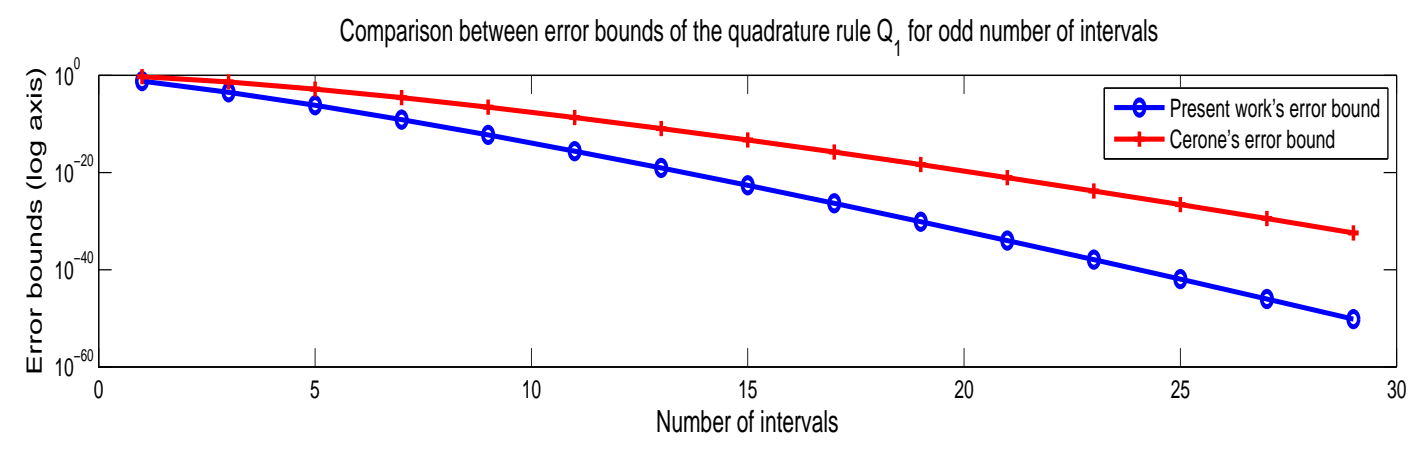

Comparison between error bounds of the quadrature rule $Q_{2}$ for odd number of intervals

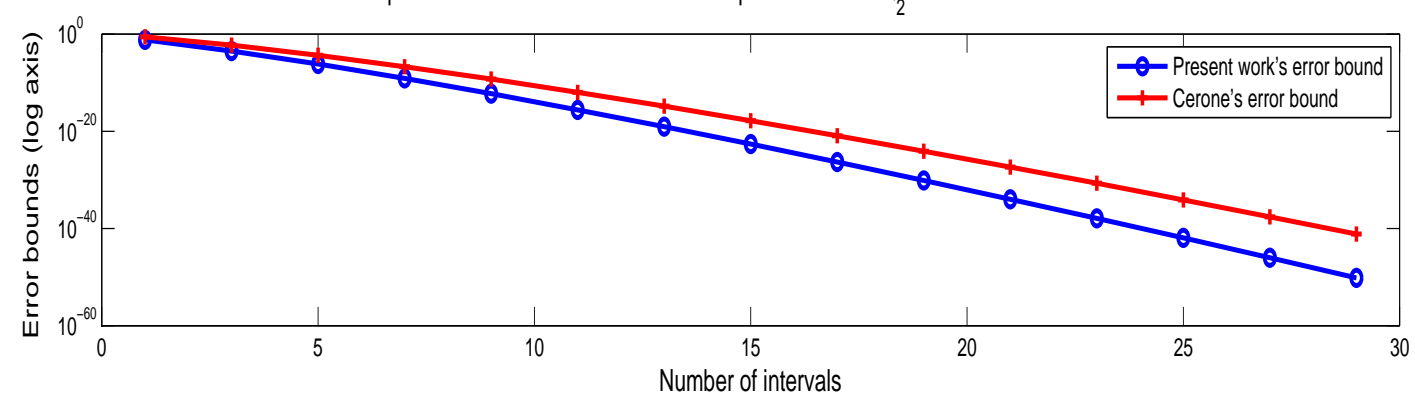

Comparison between error bounds of the quadrature rule $Q_{3}$ for odd number of intervals

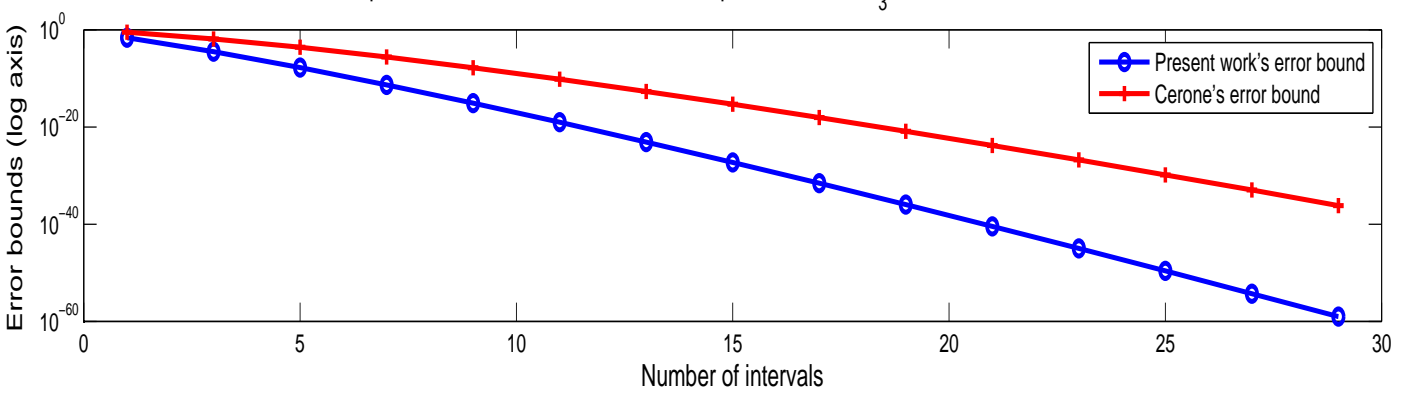

Figure 1. Comparison of Error Bounds for Odd intervals

\section{Discussion}

Cerone et al. [6] developed error bounds using 2-step kernel. But in our case, we developed error bounds with the help of 5 -step kernel. In fig. $1 \& 2$, we established a comparison between error bounds $E_{1}, E_{2}$ and $E_{3}$ for odd and even number of intervals. These figures show that our error bounds are smaller than the error bounds of Cerone et al. [6] in both cases i.e. for even and odd number of intervals. Also, it can be seen from the graphs that the error decreases with the increase in number of intervals. Actually, use of 5-step kernel and a proper choice of scheme play a major role in minimizing the errors. It can be concluded that we have developed a very efficient new integral inequality which gives us better approximations for the quadrature. 

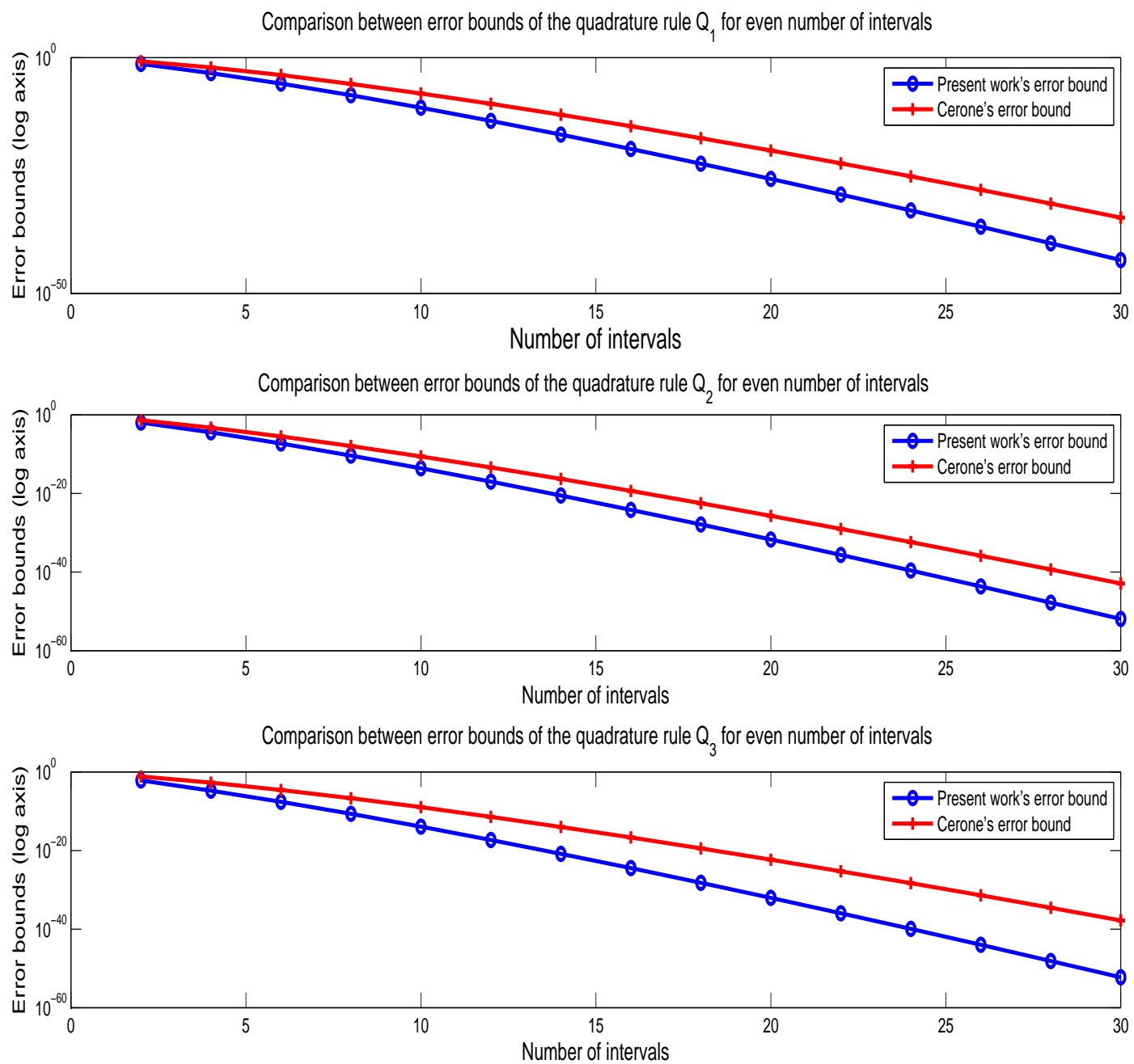

Figure 2. Comparison of Error Bounds for Even intervals

\section{REFERENCES}

[1] M. W. Alomari, A companion of ostrowski's inequality for mappings whose first derivatives are bounded and applications numerical integration, Kragujevac J. Math. 36 (2012), 77 - 82.

[2] W. G. Alshanti, A. Qayyum and M. A. Majid, Ostrowski type inequalities by using a generalized quadratic kernel, J. Inequal. Spec. Funct. 8 (4) (2017), 111-135.

[3] W. G. Alshanti and A. Qayyum, A note on new Ostrowski type inequalities using a generalized kernel, Bull. Math. Anal. Appl. 9 (1) (2017), 74-91.

[4] N. S. Barnett, S. S. Dragomir and I. Gomma, A companion for the Ostrowski and the generalized trapezoid inequalities, J. Math. Comput. Model. 50 (2009), 179-187. 
[5] H. Budak, M. Z. Sarikaya and A. Qayyum, Improvement in Companion of Ostrowski Type Inequalities for Mappings Whose First Derivatives are of Bounded Variation and Applications, Filomat 31 (2017), 5305-5314.

[6] P. Cerone, S. S. Dragomir, J. Roumeliotis and J. Sunde, A new generalization of the trapezoid formula for n-time differentiable mappings and applications, Demonstr. Math. 33(4) (2000), 719-736.

[7] S. S. Dragomir, Some companions of Ostrowski's inequality for absolutely continuous functions and applications, Bull. Korean Math. Soc. 40 (2) (2005), 213-230.

[8] S. S. Dragomir and S. Wang, An inequality of Ostrowski-Grüss type and its applications to the estimation of error bounds for some special means and for some numerical quadrature rules, Comput. Math. Appl. 33 (11) (1997), 15-20.

[9] A. Guessab and G. Schmeisser, Sharp integral inequalities of the Hermite-Hadamard type, J. Approx. Theory, 115 (2) (2002), 260-288.

[10] Z. Liu, Some companions of an Ostrowski type inequality and applications, J. Inequal. Pure Appl. Math. 10 (2) (2009), $10-12$.

[11] W. Liu, New Bounds for the Companion of Ostrowski's Inequality and Applications, Filomat, 28 (2014), $167-178$.

[12] W. Liu, Y. Zhu and J. Park, Some companions of perturbed Ostrowski-type inequalities based on the quadratic kernel function with three sections and applications, J. Inequal. Appl. 2013 (2013), Article ID 226.

[13] D. S. Mitrinvić, J. E. Pecarić and A. M. Fink, Classical and New Inequalities in Analysis, Kluwer Academic Publishers, Dordrecht, 1993.

[14] D. S. Mitrinović, J. E. Pecarić and A. M. Fink, Inequalities involving functions and their integrals and derivatives, Mathematics and its Applications. (East European Series), Kluwer Acadamic Publications Dordrecht, 1991.

[15] A. Ostrowski, Über die Absolutabweichung einer differentienbaren Funktionen von ihren Integralimittelwert, Comment. Math. Hel. 10 (1938), 226-227.

[16] A. Qayyum and S. Hussain, A new generalized Ostrowski Grüss type inequality and applications, Appl. Math. Lett. 25 (2012), 1875-1880.

[17] A. Qayyum, M. Shoaib and I. Faye, Companion of Ostrowski-type inequality based on 5-step quadratic kernel and applications, J. Nonlinear Sci. Appl. 9 (2016), 537-552.

[18] A. Qayyum, M. Shoaib and I. Faye, On new refinements and applications of efficient quadrature rules using $n$-times differentiable mappings, J. Comput. Anal. Appl. 23 (4) (2017), 723-739.

[19] A. Qayyum, A weighted Ostrowski Grüss Type Inequality for Twice Differentiable Mappings belongs to $\mathrm{L}_{p}[a, b]$ and Applications, The Proceedings of International Conference of Engineering Mathematics, London, U.K., 1-3 July (2009).

[20] N. UJević, New bounds for the first inequality of Ostrowski-Grüss type and applications, Comput. Math. Appl. 46 (2003), 421-427. 